\title{
Oral Cancer - Screening and Prevention
}

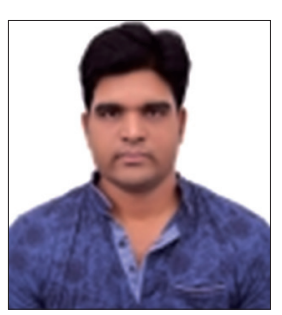

In the digital era of the $21^{\text {st }}$ century, where computer and humans are working together in almost every fields. In this era, oral cancer and its morbidity with one of the highest mortality rates among all other malignancies is also increasing day by day, hour by hour, and minute to minute.

Basic understanding of oral cancer is minimum requirement for a clinician to at least make a proper diagnosis of it. Basically, rising of oral cancer in India is due to three very important reasons which are as follows:

- Due to high tobacco chewing and alcohol consumption

- Due to infections (like human papillomavirus) (intraoral infections) play an important role in epithelial cancer of oropharynx and tonsils

- Due to obesity and even by malnutrition.

Diagnosis of oral cancer with simple clinical oral (visual inspection) examination with adequate light and sterilized proper diagnostic instruments can be proven to be an excellent screening method for early detection of precancerous lesions.

Apart from this, other diagnostic examinations which can be used in detections of oral cancers are as follows:

Oral cytology - Nowadays used for the detection of oral cancers and it helps to distinguish between benign and malignant oral lesions.

These cytological (histological) diagnoses if used can be classified as follows: ${ }^{[1]}$

- NILM (negative for intraepithelial lesion or malignancy),

- LSIL (low-grade squamous intraepithelial lesion),

- HSIL (high-grade squamous intraepithelial lesion), or

- Squamous cell carcinoma.

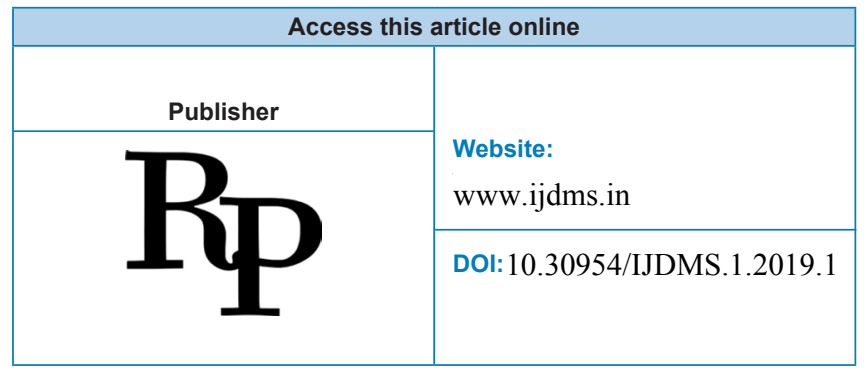

Brush biopsy (oral):

Other screening examinations which can be used in detections of oral precancerous lesions are as follows:

- Toluidine blue staining

- Autofluorescence spectroscopy

- Chemiluminescent.

- VEL (fluorescence visualization) scope oral screening - it is non-invasive oral cancer screening device which primarily focuses on wireless handheld scope to visualize oral mucosal abnormalities (e.g., hyperkeratosis, hyperchromatin, and increased cellular/nuclear pleomorphism) and metabolism (e.g., concentration of flavin adenine dinucleotide and nicotinamide adenine dinucleotide) of the epithelium, as well as changes of the subepithelial stroma (e.g., composition of collagen matrix and elastin), alter their interaction with light. ${ }^{[2]}$

Prevention of oral cancer can only done by taken strictly measures as follows:

Reduction in tobacco chewing (world's most avoidable cause) which can be only done by camps, use of social media, increase in taxation, and also community mobilization.

\section{REFERENCES}

1. Sekine J, Nakatani E, Hideshima K, Iwahashi T, Sasaki H. Diagnostic accuracy of oral cancer cytology in a pilot study. Diagn Pathol 2017;12:27.

2. Fedele S. Diagnostic aids in the screening of oral cancer. Head Neck Oncol 2009;1:5.

Anand Kumar

Department of Dentistry, Moti Lal Nehru Medical College, Prayagraj, Uttar Pradesh, India

Address for Correspondence:

Dr. Anand Kumar,

Department of Dentistry, Moti Lal Nehru

Medical College, Prayagraj, Uttar Pradesh, India.

E-mail: anandkmr901@gmail.com

How to cite this article: Kumar A. Oral Cancer - Screening and Prevention. Int J Dent Med Spec 2019;6(1):1.

Source of Support: None; Conflict of Interest: None 